\title{
An Iterative Scheme for Solving Systems of Nonlinear Fredholm Integrodifferential Equations
}

\author{
M. I. Berenguer, D. Gámez, and A. J. López Linares \\ Departamento de Matemática Aplicada, E.T.S. Ingeniera Edificación, Universidad de Granada, C/Severo Ochoa s/n, \\ 18071 Granada, Spain
}

Correspondence should be addressed to D. Gámez; domingo@ugr.es

Received 21 February 2014; Revised 28 May 2014; Accepted 6 June 2014; Published 6 July 2014

Academic Editor: Ahmet Yasar Ozban

Copyright (C) 2014 M. I. Berenguer et al. This is an open access article distributed under the Creative Commons Attribution License, which permits unrestricted use, distribution, and reproduction in any medium, provided the original work is properly cited.

Using fixed-point techniques and Faber-Schauder systems in adequate Banach spaces, we approximate the solution of a system of nonlinear Fredholm integrodifferential equations of the second kind.

\section{Introduction}

An important area of research interest is the study of systems of nonlinear Fredholm integrodifferential equations. A system of nonlinear Fredholm integrodifferential equations can be written in vectorial form as

$$
\begin{gathered}
\mathbf{X}^{\prime}(t)=\mathbf{F}(t, \mathbf{X}(t))+\int_{0}^{1} \mathbf{K}(t, s, \mathbf{X}(s)) d s \\
(t, s \in[0,1]), \\
\mathbf{X}(0)=\boldsymbol{\rho},
\end{gathered}
$$

where $\mathbf{X}=\left[x_{1}, \ldots, x_{n}\right]^{T} \in C\left([0,1], \mathbb{R}^{n}\right)$ is the solution to be calculated and $\mathbf{F}=\left[F_{1}, \ldots, F_{n}\right]^{T} \in C\left([0,1] \times \mathbb{R}^{n}, \mathbb{R}^{n}\right), \mathbf{K}=$ $\left[K_{1}, \ldots, K_{n}\right]^{T} \in C\left([0,1]^{2} \times \mathbb{R}^{n}, \mathbb{R}^{n}\right)$, and $\boldsymbol{\rho}=\left[\rho_{1}, \ldots, \rho_{n}\right]^{T} \in$ $\mathbb{R}^{n}$ are known.

Observe that, for $i=1, \ldots, n$, the $i$ th equation of the system (1) adopts the form

$$
\begin{aligned}
x_{i}^{\prime}(t)= & F_{i}\left(t, x_{1}(t), \ldots, x_{n}(t)\right) \\
& +\int_{0}^{1} K_{i}\left(t, s, x_{1}(s), \ldots, x_{n}(s)\right) d s,
\end{aligned}
$$

with $x_{i}(0)=\rho_{i}$.
The system (1) is linear when for all $t, s \in[0,1]$ and $\mathbf{u}=$ $\left[u_{1}, \ldots, u_{n}\right]^{T} \in \mathbb{R}^{n}$ we have that

$$
\begin{aligned}
& \mathbf{K}(t, s, \mathbf{u})=\left[\begin{array}{ccc}
K_{11}(t, s) & \cdots & K_{1 n}(t, s) \\
\vdots & \ddots & \vdots \\
K_{n 1}(t, s) & \cdots & K_{n n}(t, s)
\end{array}\right]\left[\begin{array}{c}
u_{1} \\
\vdots \\
u_{n}
\end{array}\right], \\
& \text { with } K_{i j} \in C\left([0,1]^{2}, \mathbb{R}\right) \text {. }
\end{aligned}
$$

Many problems of physics and engineering lead to the solution of integro or integrodifferential equations or systems of such equations. In most cases, these cannot be solved by direct methods, and this, together with the powerful computer tools available, has led to the development of numerical methods that allow obtaining approximate solutions of these equations or systems of equations. In literature it is easy to find many of them.

Danfu and Xufeng [1] utilize the CAS wavelet operational matrix of integration for obtaining numerical solution of linear Fredholm integrodifferential equations. Jafarian and Measoomy Nia [2] offer an architecture of artificial neural networks (NNs) for finding approximate solution of linear Fredholm integral equations system of the second kind. In [3], Maleknejad et al. present a rationalized Haar functions method for solving linear Fredholm integrodifferential systems. In [4] Maleknejad and Tavassoli Kajani use the hybrid Legendre and block-pulse functions on interval $[0,1)$ to solve the systems of linear integrodifferential equations. In [5], 
a fully discrete version of a piecewise polynomial collocation method is constructed to solve initial or boundary value problems of linear Fredholm integrodifferential equations with weakly singular kernels. In [6], Pour-Mahmoud et al. extend the Tau method for the numerical solution of integrodifferential equations system (IDES). Yalçinbaş et al. [7] present a Legendre collocation matrix method to solve highorder linear Fredholm integrodifferential equations under the mixed conditions in terms of Legendre polynomials. Yusufoğlu in [8] introduce a numerical method for solving initial value problems for a system of integrodifferential equations (the main idea is based on the interpolations of unknown functions at distinct interpolation points). Yüzbaşı et al. [9] present a numerical matrix method based on collocation points for the approximate solution of the systems of high-order linear Fredholm integrodifferential equations with variable coefficients under mixed conditions in terms of the Bessel polynomials. Zarebnia and Ali Abadi [10] use the Sinc-collocation method to solve systems of nonlinear second-order integrodifferential equations. Berenguer et al. used in [11-14] von Neumann series, fixed-point techniques, and Faber-Schauder systems in Banach spaces to solve integro and integrodifferential equations.

In the present paper we approximate the solution of (1) and we extend the numerical approximation method given in [14]. This paper is organized as follows. In Section 2 we describe the proposed method and in Section 3 the convergence of the proposed method is investigated. In Section 4 some numerical examples are presented to show the efficiency of the proposed scheme. Finally, in Section 5, we end with some conclusions.

\section{Description of the Proposed Method}

We suppose that $\mathbf{F}$, $\mathbf{K}$ satisfy a global Lipschitz condition in its last variable; that is, there exist $L_{F}, L_{K}>0$ such that for all $t, s \in \mathbb{R}$ and $\mathbf{u}, \mathbf{v} \in \mathbb{R}^{n}$

$$
\begin{gathered}
\|\mathbf{F}(t, \mathbf{u})-\mathbf{F}(t, \mathbf{v})\|_{\infty} \leq L_{F}\|\mathbf{u}-\mathbf{v}\|_{\infty}, \\
\|\mathbf{K}(t, s, \mathbf{u})-\mathbf{K}(t, s, \mathbf{v})\|_{\infty} \leq L_{K}\|\mathbf{u}-\mathbf{v}\|_{\infty} .
\end{gathered}
$$

If we reformulate the system (1) in terms of an adequate operator, we can derive its unique solvability under a suitable condition. To be more precise, if $\mathscr{V}: C\left([0,1], \mathbb{R}^{n}\right) \rightarrow$ $C\left([0,1], \mathbb{R}^{n}\right)$ is the operator given for each $\mathbf{X}$ as

$$
\begin{aligned}
\mathscr{V}(\mathbf{X})(\cdot):= & \boldsymbol{\rho}+\int_{0}^{(\cdot)} \mathbf{F}(u, \mathbf{X}(u)) d u \\
& +\int_{0}^{(\cdot)} \int_{0}^{1} \mathbf{K}(u, s, \mathbf{X}(s)) d s d u,
\end{aligned}
$$

then solving (1) is equivalent to finding a fixed point $\mathbf{X}$ of the operator $\mathscr{V}$.

A direct calculation over $\mathscr{V}$ leads to

$$
\|\mathscr{V}(\mathbf{Y})-\mathscr{V}(\mathbf{Z})\| \leq L\|\mathbf{Y}-\mathbf{Z}\|
$$

for all $\mathbf{Y}, \mathbf{Z} \in C\left([0,1], \mathbb{R}^{n}\right)$, where $L:=L_{F}+L_{K}$, with $L_{F}$ and $L_{K}$ being the Lipschitz constants of $\mathbf{F}$ and $\mathbf{K}$, respectively.
Thus, according to the Banach fixed-point theorem (see [15]), (5) has one unique fixed point; equivalently, (1) has one and only one solution $\mathbf{X} \in C\left([0,1], \mathbb{R}^{n}\right)$ provided that $L<1$. In addition, for each $\widetilde{\mathbf{X}} \in C\left([0,1], \mathbb{R}^{n}\right)$,

$$
\left\|\mathscr{V}^{m}(\widetilde{\mathbf{X}})-\mathbf{X}\right\| \leq \frac{L^{m}}{1-L}\|\mathscr{V}(\widetilde{\mathbf{X}})-\widetilde{\mathbf{X}}\|
$$

and in particular $\mathbf{X}=\lim _{m} \mathscr{V}^{m}(\widetilde{\mathbf{X}})$.

Then, given $\widetilde{\mathbf{X}}=\left[\widetilde{x}_{1}, \ldots, \widetilde{x}_{n}\right]^{T} \in C\left([0,1], \mathbb{R}^{n}\right)$, our next target is to obtain $\mathscr{V}^{m}(\widetilde{\mathbf{X}})$. We consider the functions $\boldsymbol{\varphi} \epsilon$ $C\left([0,1], \mathbb{R}^{n}\right)$ and $\boldsymbol{\xi} \in C\left([0,1]^{2}, \mathbb{R}^{n}\right)$ defined by

$$
\begin{gathered}
\varphi(t):=\mathbf{F}(t, \widetilde{\mathbf{X}}(t))=\left[\varphi_{1}(t), \ldots, \varphi_{n}(t)\right]^{T}, \\
\xi(t, s):=\mathbf{K}(t, s, \widetilde{\mathbf{X}}(s))=\left[\xi_{1}(t, s), \ldots, \xi_{1}(t, s)\right]^{T} .
\end{gathered}
$$

Observe that $\mathscr{V}(\widetilde{\mathbf{X}})(t)=\left[(\mathscr{V}(\widetilde{\mathbf{X}}))_{1}(t), \ldots,(\mathscr{V}(\widetilde{\mathbf{X}}))_{n}(t)\right]^{T}$, where for all $i=1, \ldots, n$

$$
(\mathscr{V}(\widetilde{\mathbf{X}}))_{i}(t)=\rho_{i}+\int_{0}^{t} \varphi_{i}(u) d u+\int_{0}^{t} \int_{0}^{1} \xi_{i}(u, s) d s d u
$$

Now we will make use of the usual Schauder basis $\left\{b_{i}\right\}_{i \geq 1}$ in $C([0,1], \mathbb{R})$ and the usual Schauder basis $\left\{B_{i}\right\}_{i \geq 1}$ for the Banach space $C\left([0,1]^{2}, \mathbb{R}\right)$ (see $[16,17]$ ), although the numerical method given works equally well by replacing it with any complete biorthogonal system in this space. We denote by $\left\{P_{i}\right\}_{i \geq 1}$ and $\left\{Q_{i}\right\}_{i \geq 1}$ the sequences of projections in $C([0,1], \mathbb{R})$ and $C\left([0,1]^{2}, \mathbb{R}\right)$, respectively (see Section 3 in [11]).

Then, for all $t \in[0,1]$ and $i=1, \ldots, n$,

$$
\begin{aligned}
(\mathscr{V}(\widetilde{\mathbf{X}}))_{i}(t)= & \rho_{i}+\sum_{k \geq 1} \lambda_{i k} \int_{0}^{t} b_{k}(u) d u \\
& +\sum_{k \geq 1} \delta_{i k} \int_{0}^{t} \int_{0}^{1} B_{k}(u, s) d s d u
\end{aligned}
$$

where $\left\{\lambda_{i k}\right\}_{k \geq 1}$ and $\left\{\delta_{i k}\right\}_{k \geq 1}$ are the sequences of scalars satisfying $\varphi_{i}=\sum_{k \geq 1} \lambda_{i k} b_{k}$ and $\xi_{i}=\sum_{k \geq 1} \delta_{i k} B_{k}$, where $\lambda_{i 1}=$ $\varphi_{i}\left(t_{1}\right)$ and $\delta_{i 1}=\xi_{i}\left(t_{1}, t_{1}\right)$, and for $k \geq 2$ is $\lambda_{i k}=\varphi_{i}\left(t_{k}\right)-$ $\sum_{l=1}^{k-1} b_{l}^{*}\left(\varphi_{i}\right) b_{l}\left(t_{k}\right)$ and $\delta_{i k}=\xi_{i}\left(t_{p}, t_{q}\right)-\sum_{l=1}^{k-1} B_{l}^{*}\left(\xi_{i}\right) B_{l}\left(t_{p}, t_{q}\right)$ with $\sigma(k)=(p, q)$.

In view of (10) we can calculate, at least in a theoretical way, $\mathscr{V}^{m}(\widetilde{\mathbf{X}})$. From a practical point of view, in general these calculations are not possible explicitly, since they are infinite sums. The idea of our numerical method is to truncate them by means of the projections of the Schauder bases $\left\{b_{i}\right\}_{i \geq 1}$, $\left\{B_{i}\right\}_{i \geq 1}$ and approximate the solution in this way. Specifically, we consider the sequence $\left\{\mathbf{X}_{\mathbf{r}}\right\}_{r \geq 1}$ defined as follows. Let $\mathbf{X}_{0}(t):=\widetilde{\mathbf{X}}(t)=\left[x_{01}(t), \ldots, x_{0 n}(t)\right]^{T} \in C\left([0,1], \mathbb{R}^{n}\right)$, $t \in[0,1]$, and $\left\{i_{1}, i_{2}, \ldots\right\},\left\{j_{1}, j_{2}, \ldots\right\}$ be subsets of natural 
numbers and $m \in \mathbb{N}$. Define inductively for $r \in\{1, \ldots, m\}$ and $t, s \in[0,1]$

$$
\begin{gathered}
\mathbf{X}_{\mathbf{r}}(t)=\left[x_{r 1}(t), \ldots, x_{r n}(t)\right]^{T} \\
:=\boldsymbol{\rho}+\left[\int_{0}^{t} P_{i_{r}}\left(\psi_{r-1,1}(u)\right) d u\right. \\
\left.\ldots, \int_{0}^{t} P_{i_{r}}\left(\psi_{r-1, n}(u)\right) d u\right]^{T} \\
+\left[\int_{0}^{t} \int_{0}^{1} Q_{j_{r}^{2}}\left(\phi_{r-1,1}(u, s)\right) d s d u,\right. \\
\left.\ldots, \int_{0}^{t} \int_{0}^{1} Q_{j_{r}^{2}}\left(\phi_{r-1, n}(u, s)\right) d s d u\right]^{T},
\end{gathered}
$$

where

$$
\begin{gathered}
\Psi_{\mathbf{r}-\mathbf{1}}(t)=\left[\psi_{r-1,1}(t), \ldots, \psi_{r-1, n}(t)\right]^{T} \\
:=\mathbf{F}\left(t, \mathbf{X}_{\mathbf{r}-\mathbf{1}}(t)\right), \\
\psi_{r-1, i}(t)=F_{i}\left(t, \mathbf{X}_{\mathbf{r}-\mathbf{1}}(t)\right) \quad \text { for } i=1, \ldots, n, \\
\Phi_{\mathbf{r}-\mathbf{1}}(t, s)=\left[\phi_{r-1,1}(t, s), \ldots, \phi_{r-1, n}(t, s)\right]^{T} \\
:=\mathbf{K}\left(t, s, \mathbf{X}_{\mathbf{r}-\mathbf{1}}(s)\right), \\
\phi_{r-1, i}(t, s)=K_{i}\left(t, s, \mathbf{X}_{\mathbf{r}-\mathbf{1}}(s)\right) \quad \text { for } i=1, \ldots, n .
\end{gathered}
$$

Observe that for $i=1, \ldots, n$

$$
\begin{aligned}
x_{r i}(t)= & \rho_{i}+\int_{0}^{t} P_{i_{r}}\left(\psi_{r-1, i}(u)\right) d u \\
& +\int_{0}^{t} \int_{0}^{1} Q_{j_{r}^{2}}\left(\phi_{r-1, i}(u, s)\right) d s d u .
\end{aligned}
$$

\section{Convergence of the Scheme}

This section is devoted to provide a convergence analysis for the numerical scheme $\left\{\mathbf{X}_{\mathbf{r}}\right\}_{r \geq 1}$. To analyze the convergence we employ the following two results.

Theorem 1. Let $\mathbf{F} \in C^{1}\left([0,1] \times \mathbb{R}^{n}, \mathbb{R}^{n}\right)$ and $\mathbf{K} \in C^{1}\left([0,1]^{2} \times\right.$ $\left.\mathbb{R}^{n}, \mathbb{R}^{n}\right)$ such that $\mathbf{F}, \mathbf{K}$ and $\partial F_{i} / \partial t, \partial F_{i} / \partial u_{j}, \partial K_{i} / \partial t, \partial K_{i} / \partial s$, and $\partial K_{i} / \partial u_{j}$ for each $i, j=1, \ldots, n$ satisfy a global Lipschitz condition in the last variables. Then, maintaining the notation above, the sequences $\left\{\psi_{r-1, i}^{\prime}\right\}_{r \geq 1},\left\{\partial \phi_{r-1, i} / \partial t\right\}_{r \geq 1}$, and $\left\{\partial \phi_{r-1, i} / \partial s\right\}_{r \geq 1}$, with $i=1, \ldots, n$, are bounded.

Proof. Let us fix $i=1, \ldots, n$ and write $\partial F_{i} / \partial \mathbf{u}=\left[\partial F_{i} / \partial u_{1}, \ldots\right.$, $\left.\partial F_{i} / \partial u_{n}\right]^{T}, \partial K_{i} / \partial \mathbf{u}=\left[\partial K_{i} / \partial u_{1}, \ldots, \partial K_{i} / \partial u_{n}\right]^{T}$. Making use of definitions (11), it follows that, for all $r \geq 1, i=1, \ldots, n$, and $t, s \in[0,1]$,

$$
\begin{gathered}
\psi_{r-1, i}^{\prime}(t)=\frac{\partial F_{i}}{\partial t}\left(t, \mathbf{X}_{\mathbf{r}-\mathbf{1}}(t)\right)+\frac{\partial F_{i}}{\partial \mathbf{u}}\left(t, \mathbf{X}_{\mathbf{r}-\mathbf{1}}(t)\right) \cdot \mathbf{X}_{\mathbf{r}-\mathbf{1}}^{\prime}(t), \\
\frac{\partial \phi_{r-1, i}}{\partial t}(t, s)=\frac{\partial K_{i}}{\partial t}\left(t, s, \mathbf{X}_{\mathbf{r}-\mathbf{1}}(s)\right), \\
\frac{\partial \phi_{r-1, i}}{\partial s}(t, s)=\frac{\partial K_{i}}{\partial s}\left(t, s, \mathbf{X}_{\mathbf{r}-\mathbf{1}}(s)\right) \\
+\frac{\partial K_{i}}{\partial \mathbf{u}}\left(t, s, \mathbf{X}_{\mathbf{r}-\mathbf{1}}(s)\right) \cdot \mathbf{X}_{\mathbf{r}-\mathbf{1}}^{\prime}(s),
\end{gathered}
$$

where "." stands for the usual inner product in $\mathbb{R}^{n}$.

For all $r \geq 1$ and $t, s \in[0,1]$, we have

$$
\left\|\Psi_{\mathbf{r}-\mathbf{1}}(t)\right\|_{\infty}=\left\|\mathbf{F}\left(t, \mathbf{X}_{\mathbf{r}-\mathbf{1}}(t)\right)\right\|_{\infty}
$$

$$
\begin{aligned}
& \leq\left\|\mathbf{F}\left(t, \mathbf{X}_{\mathbf{r}-\mathbf{1}}(t)\right)-\mathbf{F}(t, \mathbf{0})\right\|_{\infty}+\|\mathbf{F}(t, \mathbf{0})\|_{\infty} \\
& \leq L_{F}\left\|\mathbf{X}_{\mathbf{r}-\mathbf{1}}(t)\right\|_{\infty}+R_{F},
\end{aligned}
$$

with $R_{F}:=\max _{t \in[0,1]}\|\mathbf{F}(t, \mathbf{0})\|_{\infty}$ and $L_{F}$ being the Lipschitz constant of $\mathbf{F}$ and analogous

$$
\begin{aligned}
\left\|\boldsymbol{\Phi}_{\mathbf{r}-\mathbf{1}}(t, s)\right\|_{\infty}= & \left\|\mathbf{K}\left(t, s, \mathbf{X}_{\mathbf{r}-\mathbf{1}}(s)\right)\right\|_{\infty} \\
\leq & \left\|\mathbf{K}\left(t, s, \mathbf{X}_{\mathbf{r}-\mathbf{1}}(s)\right)-\mathbf{K}(t, s, \mathbf{0})\right\|_{\infty} \\
& +\|\mathbf{K}(t, s, \mathbf{0})\|_{\infty} \\
\leq & L_{K}\left\|\mathbf{X}_{\mathbf{r}-\mathbf{1}}(s)\right\|_{\infty}+R_{K},
\end{aligned}
$$

with $R_{K}:=\max _{(t, s) \in[0,1]^{2}}\|\mathbf{K}(t, s, \mathbf{0})\|_{\infty}$ and $L_{K}$ being the Lipschitz constant of $\mathbf{K}$.

Now we will show that the sequence $\left\{\mathbf{X}_{\mathbf{r}}\right\}_{r \geq 1}$ is bounded.

From the monotonicity of the Schauder bases $\left\{b_{i}\right\}_{i \geq 1}$, $\left\{B_{i}\right\}_{i \geq 1}$ and the recursive application of this inequality and the following one,

$$
\begin{aligned}
\left\|\mathbf{X}_{\mathbf{r}}(t)\right\|_{\infty} \leq & \|\rho\|_{\infty}+\int_{0}^{t}\left\|\Psi_{\mathbf{r}-\mathbf{1}}(u)\right\|_{\infty} d u \\
& +\int_{0}^{t} \int_{0}^{1}\left\|\Phi_{r-1}(u, s)\right\|_{\infty} d s d u,
\end{aligned}
$$

we have

$$
\begin{aligned}
\left\|\mathbf{X}_{\mathbf{r}}(t)\right\|_{\infty} \leq & \|\boldsymbol{\rho}\|_{\infty}+\int_{0}^{t}\left(L_{F}\left\|\mathbf{X}_{\mathbf{r}-\mathbf{1}}(u)\right\|_{\infty}+R_{F}\right) d u \\
& +\int_{0}^{t} \int_{0}^{1}\left(L_{K}\left\|\mathbf{X}_{\mathbf{r}-\mathbf{1}}(s)\right\|_{\infty}+R_{K}\right) d s d u \\
\leq & \Gamma+L\left\|\mathbf{X}_{\mathbf{r}-\mathbf{1}}\right\|,
\end{aligned}
$$


with $L:=L_{F}+L_{K}, \Gamma:=\|\rho\|_{\infty}+R$ and $R:=R_{F}+R_{K}$. Applying it inductively, we arrive at

$$
\left\|\mathbf{X}_{\mathbf{r}}\right\| \leq \Gamma \sum_{k=1}^{r} L^{k-1}+L^{r}\left\|\mathbf{X}_{\mathbf{0}}\right\|,
$$

for all $r \geq 1$, and therefore the sequence $\left\{\mathbf{X}_{\mathbf{r}}\right\}_{r \geq 1}$ is bounded.

Since the sequence $\left\{\mathbf{X}_{\mathbf{r}}\right\}_{r>1}$ in (15) and (16) is bounded it follows that $\left\{\boldsymbol{\Psi}_{r-1}\right\}_{r \geq 1}$ and $\left\{\boldsymbol{\Phi}_{r-1}\right\}_{r \geq 1}$ are uniformly bounded.

For $i=1, \ldots, n$, we have

$$
\begin{aligned}
\mid \frac{\partial F_{i}}{\partial t} & \left(t, \mathbf{X}_{\mathbf{r}-\mathbf{1}}(t)\right) \mid \\
& \leq\left|\frac{\partial F_{i}}{\partial t}\left(t, \mathbf{X}_{\mathbf{r}-\mathbf{1}}(t)\right)-\frac{\partial F_{i}}{\partial t}(t, \mathbf{0})\right|+\left|\frac{\partial F_{i}}{\partial t}(t, \mathbf{0})\right| \\
& \leq L_{F_{i}}\left\|\mathbf{X}_{\mathbf{r}-\mathbf{1}}\right\|+R_{i},
\end{aligned}
$$

with $R_{i}:=\max _{t \in[\alpha, \alpha+\beta]}\left|\left(\partial F_{i} / \partial t\right)(t, \mathbf{0})\right|$ and $L_{F_{i}}$ as the Lipschitz constant of $\partial F_{i} / \partial t$.

Meanwhile,

$$
\begin{aligned}
& \left\|\frac{\partial F_{i}}{\partial \mathbf{u}}\left(t, \mathbf{X}_{\mathbf{r}-\mathbf{1}}(t)\right)\right\|_{\infty} \\
& \quad \leq\left\|\frac{\partial F_{i}}{\partial \mathbf{u}}\left(t, \mathbf{X}_{\mathbf{r}-\mathbf{1}}(t)\right)-\frac{\partial F_{i}}{\partial \mathbf{u}}(t, \mathbf{0})\right\|_{\infty}+\left\|\frac{\partial F_{i}}{\partial \mathbf{u}}(t, \mathbf{0})\right\|_{\infty} \\
& \quad \leq L_{F_{i}}^{*}\left\|\mathbf{X}_{\mathbf{r}-\mathbf{1}}\right\|+R_{i}^{*},
\end{aligned}
$$

with $R_{i}^{*}:=\max _{j=1, \ldots, n} \max _{t \in[\alpha, \alpha+\beta]}\left|\left(\partial F_{i} / \partial u_{j}\right)(t, \mathbf{0})\right|$ and $L_{F_{i}}^{*}$ as the maximum of the Lipschitz constants for each $\partial F_{i} / \partial u_{j}, j=$ $1, \ldots, n$.

Therefore, $\quad\left\{\left(\partial F_{i} / \partial t\right)\left(t, \mathbf{X}_{\mathbf{r}-\mathbf{1}}(t)\right)\right\}_{r \geq 1} \quad$ and $\quad\left\{\left(\partial F_{i} / \partial \mathbf{u}\right)(t\right.$, $\left.\left.\mathbf{X}_{\mathbf{r}-\mathbf{1}}(t)\right)\right\}_{r \geq 1}$ are bounded.

Next, we will show that the sequence $\left\{\mathbf{X}_{\mathbf{r}-1}^{\prime}\right\}_{r \geq 1}$ is bounded.

Given $r \geq 1$, taking into account the definition of $\mathbf{X}_{\mathbf{r}}$, we have for all $t \in[0,1]$ that

$$
\begin{array}{r}
\mathbf{X}_{\mathbf{r}}^{\prime}(t)=\left[P_{i_{r}}\left(\psi_{r-1,1}(t)\right), \ldots, P_{i_{r}}\left(\psi_{r-1, n}(t)\right)\right]^{T} \\
+\left[\int_{0}^{1} Q_{j_{r}^{2}}\left(\phi_{r-1,1}(t, s)\right) d s,\right. \\
\left.\quad \ldots, \int_{0}^{1} Q_{j_{r}^{2}}\left(\phi_{r-1, n}(t, s)\right) d s\right]^{T} .
\end{array}
$$

In view of the monotonicity of the Schauder bases $\left\{b_{i}\right\}_{i \geq 1}$ and $\left\{B_{i}\right\}_{i>1}$ and (15), (16), and (19), we obtain

$$
\begin{aligned}
\left\|\mathbf{X}_{\mathbf{r}}^{\prime}\right\| & \leq\left\|\boldsymbol{\Psi}_{\mathbf{r}-1}\right\|+\left\|\Phi_{\mathbf{r}-1}\right\| \\
& \leq L_{F}\left\|\mathbf{X}_{\mathbf{r}-1}\right\|+R_{F}+L_{K}\left\|\mathbf{X}_{\mathbf{r}-1}\right\|+R_{K} \\
& \leq\left\|\mathbf{X}_{\mathbf{r}-1}\right\|\left(L_{F}+L_{K}\right)+R_{F}+R_{K} \\
& \leq \Gamma \sum_{k=1}^{r} L^{k}+L^{r}\left\|\mathbf{X}_{\mathbf{0}}\right\|+R .
\end{aligned}
$$

Therefore, the sequence $\left\{\mathbf{X}_{\mathbf{r}}^{\prime}\right\}_{r \geq 1}$ is also bounded.

We will prove that the sequences $\left\{\left(\partial K_{i} / \partial t\right)(t, s\right.$, $\left.\left.\mathbf{X}_{\mathbf{r}-\mathbf{1}}(s)\right)\right\}_{r \geq 1},\left\{\left(\partial K_{i} / \partial s\right)\left(t, s, \mathbf{X}_{\mathbf{r}-\mathbf{1}}(s)\right)\right\}_{r \geq 1}$, and $\left\{\left(\partial K_{i} / \partial \mathbf{u}\right)(t, s\right.$, $\left.\left.\mathbf{X}_{\mathbf{r}-1}(s)\right)\right\}_{r \geq 1}$ are bounded.

For $i=1, \ldots, n$, we have

$$
\begin{aligned}
& \left|\frac{\partial K_{i}}{\partial t}\left(t, s, \mathbf{X}_{\mathbf{r}-\mathbf{1}}(s)\right)\right| \\
& \quad \leq\left|\frac{\partial K_{i}}{\partial t}\left(t, s, \mathbf{X}_{\mathbf{r}-\mathbf{1}}(s)\right)-\frac{\partial K_{i}}{\partial t}(t, s, \mathbf{0})\right|+\left|\frac{\partial K_{i}}{\partial t}(t, s, \mathbf{0})\right| \\
& \quad \leq M_{1 i}\left\|\mathbf{X}_{\mathbf{r}-\mathbf{1}}\right\|+N_{1 i},
\end{aligned}
$$

with $N_{1 i}:=\max _{(t, s) \in[0,1]^{2}}\left|\left(\partial K_{i} / \partial t\right)(t, s, \mathbf{0})\right|$ and $M_{1 i}$ as the Lipschitz constant of $\partial K_{i} / \partial t$.

By repeating the previous argument we obtain

$$
\left|\frac{\partial K_{i}}{\partial s}\left(t, s, \mathbf{X}_{\mathbf{r}-\mathbf{1}}(s)\right)\right| \leq M_{2 i}\left\|\mathbf{X}_{\mathbf{r}-\mathbf{1}}\right\|+N_{2 i},
$$

with $N_{2 i}:=\max _{(t, s) \in[0,1]^{2}}\left|\left(\partial K_{i} / \partial s\right)(t, s, \mathbf{0})\right|$ and $M_{2 i}$ as the Lipschitz constant of $\partial K_{i} / \partial s$.

Therefore, the sequences $\left\{\left(\partial K_{i} / \partial t\right)\left(t, s, \mathbf{X}_{\mathbf{r}-1}(s)\right)\right\}_{r \geq 1}$ and $\left\{\left(\partial K_{i} / \partial s\right)\left(t, s, \mathbf{X}_{\mathbf{r}-\mathbf{1}}(s)\right)\right\}_{r \geq 1}$ are bounded.

Meanwhile,

$$
\begin{aligned}
& \left\|\frac{\partial K_{i}}{\partial \mathbf{u}}\left(t, s, \mathbf{X}_{\mathbf{r}-\mathbf{1}}(s)\right)\right\|_{\infty} \\
& \quad \leq\left\|\frac{\partial K_{i}}{\partial \mathbf{u}}\left(t, s, \mathbf{X}_{\mathbf{r}-\mathbf{1}}(s)\right)-\frac{\partial K_{i}}{\partial \mathbf{u}}(t, s, \mathbf{0})\right\|_{\infty}+\left\|\frac{\partial K_{i}}{\partial \mathbf{u}}(t, s, \mathbf{0})\right\|_{\infty} \\
& \quad \leq M_{3 i}\left\|\mathbf{X}_{\mathbf{r}-1}\right\|+N_{3 i},
\end{aligned}
$$

with $N_{3 i}:=\max _{j=1, \ldots, n} \max _{(t, s) \in[0,1]^{2}}\left|\left(\partial K_{i} / \partial u_{j}\right)(t, s, \mathbf{0})\right|$ and $M_{3 i}$ as the maximum of the Lipschitz constants for each $\partial K_{i} / \partial u_{j}, j=1, \ldots, n$. Therefore, $\left\{\left(\partial K_{i} / \partial \mathbf{u}\right)\left(t, s, \mathbf{X}_{\mathbf{r}-1}(s)\right)\right\}$ is bounded.

In view of the identities (14), we have that the sequences, $\left\{\psi_{r-1, i}^{\prime}\right\}_{r \geq 1},\left\{\partial \phi_{r-1, i} / \partial t\right\}_{r \geq 1}$, and $\left\{\partial \phi_{r-1, i} / \partial s\right\}_{r \geq 1}$, with $i=$ $1, \ldots, n$, are bounded.

For a dense subset $\left\{t_{i}\right\}_{i \geq 1}$ of distinct points in $[0,1]$, let $T_{i}$ be the set $\left\{t_{j}, 1 \leq j \leq i\right\}$ ordered in an increasing way for $i \geq 2$. Let $\Delta T_{i}$ denote the maximum distance between two consecutive points of $T_{i}$.

Theorem 2. With the previous notation and the same hypothesis as in Theorem 1, for all $r \geq 1$, there are $\eta_{r}, \tau_{r}>0$ and $i_{r}, j_{r} \geq 2$ such that

$$
\begin{aligned}
& \left\|\left[\psi_{r-1,1}-P_{i_{r}}\left(\psi_{r-1,1}\right), \ldots, \psi_{r-1, n}-P_{i_{r}}\left(\psi_{r-1, n}\right)\right]^{T}\right\| \\
& \quad \leq \eta_{r} \Delta T_{i_{r}}, \\
& \left\|\left[\phi_{r-1,1}-Q_{j_{r}^{2}}\left(\phi_{r-1,1}\right), \ldots, \phi_{r-1, n}-Q_{j_{r}^{2}}\left(\phi_{r-1, n}\right)\right]^{T}\right\| \\
& \quad \leq \tau_{r} \Delta T_{j_{r}} .
\end{aligned}
$$


Proof. The announced estimation follows from the inequalities obtained in Propositions 4 and 5 in [11], respectively, and applying Theorem 1.

In the result below we show that the sequence defined in (11) approximates the solution of (1).

Theorem 3. With the same hypothesis as in Theorem 1, suppose that $\mathscr{V}: C\left([0,1], \mathbb{R}^{n}\right) \rightarrow C\left([0,1], \mathbb{R}^{n}\right)$ is the integral operator (5), $\widetilde{\mathbf{X}} \in C\left([0,1], \mathbb{R}^{n}\right)$, and that $\left\{\mathbf{X}_{\mathbf{r}}\right\}_{r \geq 1}$ is the sequence defined by (11). Let us also assume that $m \in \mathbb{N}, i_{r}, j_{r} \geq 2$, and $\left\{\varepsilon_{1}, \ldots, \varepsilon_{m}\right\}$ is a set of positive numbers such that for all $r \in\{1, \ldots, m\}$ we have

$$
\Delta T_{i_{r}} \leq \frac{\varepsilon_{r}}{2 \eta_{r}}, \quad \Delta T_{j_{r}} \leq \frac{\varepsilon_{r}}{2 \tau_{r}} .
$$

Then

$$
\left\|\mathscr{V}\left(\mathbf{X}_{\mathbf{r}-1}\right)-\mathbf{X}_{\mathbf{r}}\right\| \leq \varepsilon_{r} .
$$

Moreover, if $\mathbf{X}$ is the exact solution of the integral equation (1), then the error $\left\|\mathbf{X}-\mathbf{X}_{\mathbf{m}}\right\|$ is given by

$$
\left\|\mathbf{X}-\mathbf{X}_{\mathbf{m}}\right\| \leq \frac{L^{m}}{1-L}\|\mathscr{V} \widetilde{\mathbf{X}}-\widetilde{\mathbf{X}}\|+\sum_{r=1}^{m} L^{m-r} \varepsilon_{r} .
$$

Proof. For $m \geq 1$, from (7), we have

$$
\left\|\mathbf{X}-\mathscr{V}^{m} \widetilde{\mathbf{X}}\right\| \leq \frac{L^{m}}{1-L}\|\mathscr{V} \widetilde{\mathbf{X}}-\widetilde{\mathbf{X}}\| .
$$

First we deal with proving (29). For all $r \in\{1, \ldots, m\}$ and $t \in[0,1]$, Theorem 2 gives

$$
\begin{gathered}
\left\|\mathscr{V} \mathbf{X}_{\mathbf{r}-1}(t)-\mathbf{X}_{\mathbf{r}}(t)\right\|_{\infty} \\
=\| \boldsymbol{\rho}+\left[\int_{0}^{t} \psi_{r-1,1}(u) d u, \ldots, \int_{0}^{t} \psi_{r-1, n}(u) d u\right]^{T} \\
+\left[\int_{0}^{t} \int_{0}^{1} \phi_{r-1,1}(u, s) d s d u,\right. \\
\left.\ldots, \int_{0}^{t} \int_{0}^{1} \phi_{r-1, n}(u, s) d s d u\right]^{T} \\
-\left(\rho+\left[\int_{0}^{t} P_{i_{r}}\left(\psi_{r-1,1}(u)\right) d u,\right.\right. \\
\left.\ldots, \int_{0}^{t} P_{i_{r}}\left(\psi_{r-1, n}(u)\right) d u\right]^{T} \\
+\left[\int_{0}^{t} \int_{0}^{1} Q_{j_{r}^{2}}\left(\phi_{r-1,1}(u, s)\right) d s d u,\right. \\
\left.\left.\ldots, \int_{0}^{t} \int_{0}^{1} Q_{j_{r}^{2}}\left(\phi_{r-1, n}(u, s)\right) d s d u\right]^{T}\right) \|_{\infty}
\end{gathered}
$$

$$
\begin{gathered}
\leq \|\left[\int_{0}^{t}\left(\psi_{r-1,1}(u)-P_{i_{r}}\left(\psi_{r-1,1}(u)\right)\right) d u,\right. \\
\left.\ldots, \int_{0}^{t}\left(\psi_{r-1, n}(u)-P_{i_{r}}\left(\psi_{r-1, n}(u)\right)\right) d u\right]^{T} \|_{\infty} \\
+\|\left[\int_{0}^{t} \int_{0}^{1}\left(\phi_{r-1,1}(u, s)-Q_{j_{r}}\left(\phi_{r-1,1}(u, s)\right)\right) d s d u,\right. \\
\ldots, \int_{0}^{t} \int_{0}^{1}\left(\phi_{r-1, n}(u, s)\right. \\
\leq \int_{0}^{t} \|\left[\psi_{r-1,1}(u)-P_{i_{r}}\left(\psi_{r-1,1}(u)\right),\right. \\
\left.\ldots, \psi_{r-1, n}(u)-P_{i_{r}}\left(\psi_{r-1, n}(u)\right)\right]^{T} \|_{\infty} d u \\
+\int_{0}^{t} \int_{0}^{1} \|\left[\phi_{r-1,1}(u, s)-Q_{j_{r}^{2}}\left(\phi_{r-1,1}(u, s)\right)\right), \ldots, \\
\left.\phi_{r-1, n}(u, s)-Q_{j_{r}^{2}}\left(\phi_{r-1, n}(u, s)\right)\right]^{T} \|_{\infty} d s d u
\end{gathered}
$$

And, in turn, applying (29) and recursively (6), we obtain

$$
\begin{aligned}
\left\|\mathscr{V}^{m}(\widetilde{\mathbf{X}})-\mathbf{X}_{\mathbf{m}}\right\| & =\left\|\mathscr{V}^{m}\left(\mathbf{X}_{\mathbf{0}}\right)-\mathbf{X}_{\mathbf{m}}\right\| \\
& \leq \sum_{r=1}^{m}\left\|\mathscr{V}^{m-r+1}\left(\mathbf{X}_{\mathbf{r}-\mathbf{1}}\right)-\mathscr{V}^{m-r}\left(\mathbf{X}_{\mathbf{r}}\right)\right\| \\
& =\sum_{r=1}^{m}\left\|\mathscr{V}^{m-r} \mathscr{V}\left(\mathbf{X}_{\mathbf{r}-\mathbf{1}}\right)-\mathscr{V}^{m-r}\left(\mathbf{X}_{\mathbf{r}}\right)\right\| \\
& \leq \sum_{r=1}^{m} L^{m-r}\left\|\mathscr{V}\left(\mathbf{X}_{\mathbf{r}-\mathbf{1}}\right)-\mathbf{X}_{\mathbf{r}}\right\| \\
& \leq \sum_{r=1}^{m} L^{m-r} \varepsilon_{r} .
\end{aligned}
$$

Finally, using the triangle inequality,

$$
\left\|\mathbf{X}-\mathbf{X}_{\mathbf{m}}\right\| \leq\left\|\mathbf{X}-\mathscr{V}^{m} \widetilde{\mathbf{X}}\right\|+\left\|\mathscr{V}^{m}(\widetilde{\mathbf{X}})-\mathbf{X}_{\mathbf{m}}\right\|,
$$

the proof is complete in view of (31) and (33).

Observe that under the hypotheses of Theorem 3, by inequality (30), we have

$$
\left\|\mathbf{X}-\mathbf{X}_{\mathbf{m}}\right\| \leq \frac{L^{m}}{1-L}\|\mathscr{V} \widetilde{\mathbf{X}}-\widetilde{\mathbf{X}}\|+\frac{1-L^{m}}{1-L} \max _{r \geq 1}\left\{\varepsilon_{r}\right\} .
$$

Therefore, given $\varepsilon>0$, there exists $m \geq 1$ such that $\left\|\mathbf{X}-\mathbf{X}_{\mathbf{m}}\right\|<$ $\varepsilon$ for sufficiently small $\varepsilon_{r}$, since the points of the partition can 
be chosen in such a way that $\Delta T_{i_{r}}$ and $\Delta T_{j_{r}}$ become so close to zero as we desire and the first sum on the right hand side approach zero when $m$ increases.

Remark 4. If we consider an interval $[a, b]$, then $L=(b-$ a) $L_{F}+(b-a)^{2} L_{k}$ and the bound obtained in Theorem 3 for $\left\|\mathscr{V} \mathbf{X}_{\mathbf{r}-\mathbf{1}}(t)-\mathbf{X}_{\mathbf{r}}(t)\right\|_{\infty}$ is given by

$$
\begin{aligned}
\left\|\mathscr{V} \mathbf{X}_{\mathbf{r}-\mathbf{1}}(t)-\mathbf{X}_{\mathbf{r}}(t)\right\|_{\infty} & \leq \eta_{r} \Delta T_{i_{r}}(b-a)+\tau_{r} \Delta T_{j_{r}}(b-a)^{2} \\
& \leq \varepsilon_{r},
\end{aligned}
$$

when

$$
\Delta T_{i_{r}} \leq \frac{\varepsilon_{r}}{2 \eta_{r}(b-a)}, \quad \Delta T_{j_{r}} \leq \frac{\varepsilon_{r}}{2 \tau_{r}(b-a)^{2}} .
$$

\section{Numerical Examples}

We now turn our attention to the application of the method presented in this paper for the numerical solution of six test problems. In order to construct the Schauder basis, we consider the subset $\left\{t_{i}\right\}_{i \geq 1}$ defined by $t_{1}=0, t_{2}=1$ and for $n \in \mathbb{N} \cup\{0\}, t_{i+1}=(2 k+1) / 2^{n+1}$, if $i=2^{n}+k+1$, where $0 \leq k<2^{n}$ are integers. To define the sequence $\left\{\mathbf{X}_{\mathbf{r}}\right\}_{r \geq 1}$, we take $\mathbf{X}_{0}(t)=\boldsymbol{\rho}$ and $i_{r}=j_{r}=j$ (for all $r \geq 1$ ). We include, for different values of $j$, the absolute errors committed in some representative points of $[0,1]$ when we approximate the exact solution $\mathbf{X}(t)$ by the iteration $\mathbf{X}_{\mathbf{r}}(t)$, where $r$ is shown in each table. The algorithms associated with the numerical methods were performed using Mathematica 7. In Examples 1, 2, and $3, \mathbf{X}(t)=x(t)$ and $\mathbf{X}_{\mathbf{r}}(t)=x_{r}(t)$. In the other examples, $\mathbf{X}(t)=\left[x_{1}(t), x_{2}(t)\right]^{T}$ and $\mathbf{X}_{\mathbf{r}}(t)=\left[x_{r 1}(t), x_{r 2}(t)\right]^{T}$.

Example 1. Consider the Fredholm integrodifferential equation appearing in [1]:

$$
\begin{gathered}
x^{\prime}(t)=-2 \pi \sin (2 \pi t)-\frac{1}{2} \sin (4 \pi t) \\
+\int_{0}^{1} \sin (4 \pi t+2 \pi s) y(s) d s \\
x(0)=1
\end{gathered}
$$

whose exact solution is $x(t)=\cos (2 \pi t)$. Numerical results obtained for this problem when we apply the method described in this paper and the results obtained in [1] are given in Table 1 .

Example 2. Consider the Fredholm integrodifferential equation:

$$
\begin{gathered}
x^{\prime}(t)=f(t)+\frac{1}{125} \int_{0}^{5} s t x(s) d s, \\
x(0)=\frac{1}{125},
\end{gathered}
$$

where $f(t)$ is chosen so that the exact solution is given by $x(t)=e^{-5 t} / 250$. The numerical results are given in Table 2 .
TABLE 1: Absolute errors for Example 1.

\begin{tabular}{lcc}
\hline$t$ & $\begin{array}{c}j=33 \\
\left|x_{4}(t)-x(t)\right|\end{array}$ & $\begin{array}{c}\text { Method in [1] } \\
\text { with } k=4, M=1\end{array}$ \\
\hline 0.1 & $4.93 \times 10^{-4}$ & $2.40 \times 10^{-3}$ \\
0.2 & $8.90 \times 10^{-4}$ & $5.07 \times 10^{-3}$ \\
0.3 & $4.00 \times 10^{-3}$ & $6.25 \times 10^{-3}$ \\
0.4 & $5.70 \times 10^{-3}$ & $3.87 \times 10^{-3}$ \\
0.5 & $6.40 \times 10^{-3}$ & $1.74 \times 10^{-2}$ \\
0.6 & $3.72 \times 10^{-3}$ & $1.58 \times 10^{-2}$ \\
0.7 & $7.78 \times 10^{-4}$ & $8.41 \times 10^{-3}$ \\
0.8 & $8.96 \times 10^{-4}$ & $9.65 \times 10^{-3}$ \\
0.9 & $9.40 \times 10^{-4}$ & $9.49 \times 10^{-3}$ \\
\hline
\end{tabular}

TABLE 2: Absolute errors for Example 2.

\begin{tabular}{llll}
\hline$t$ & \multicolumn{1}{c}{$j=33$} & $t$ & $j=33$ \\
& $\left|x_{3}(t)-x(t)\right|$ & \multicolumn{1}{c}{$t$} & $\left|x_{3}(t)-x(t)\right|$ \\
\hline 0.125 & $1.91 \times 10^{-3}$ & 2.625 & $5.93 \times 10^{-3}$ \\
0.250 & $2.41 \times 10^{-3}$ & 2.750 & $6.11 \times 10^{-3}$ \\
0.375 & $3.51 \times 10^{-3}$ & 2.825 & $6.29 \times 10^{-3}$ \\
0.500 & $3.83 \times 10^{-3}$ & 3.00 & $6.49 \times 10^{-3}$ \\
0.625 & $4.02 \times 10^{-3}$ & 3.125 & $6.69 \times 10^{-3}$ \\
0.750 & $4.15 \times 10^{-3}$ & 3.250 & $6.91 \times 10^{-3}$ \\
0.825 & $4.25 \times 10^{-3}$ & 3.375 & $7.13 \times 10^{-3}$ \\
1.00 & $4.34 \times 10^{-3}$ & 3.500 & $7.35 \times 10^{-3}$ \\
1.125 & $4.43 \times 10^{-3}$ & 3.625 & $7.59 \times 10^{-3}$ \\
1.250 & $4.50 \times 10^{-3}$ & 3.750 & $7.84 \times 10^{-3}$ \\
1.375 & $4.60 \times 10^{-3}$ & 3.875 & $8.09 \times 10^{-3}$ \\
1.500 & $4.69 \times 10^{-3}$ & 4 & $8.35 \times 10^{-3}$ \\
1.625 & $4.81 \times 10^{-3}$ & 4.125 & $8.62 \times 10^{-3}$ \\
1.750 & $4.91 \times 10^{-3}$ & 4.250 & $8.90 \times 10^{-3}$ \\
1.875 & $5.50 \times 10^{-3}$ & 4.375 & $9.19 \times 10^{-3}$ \\
2 & $5.51 \times 10^{-3}$ & 4.500 & $9.48 \times 10^{-3}$ \\
2.125 & $5.53 \times 10^{-3}$ & 4.625 & $9.78 \times 10^{-3}$ \\
2.250 & $5.54 \times 10^{-3}$ & 4.750 & $1.01 \times 10^{-2}$ \\
2.375 & $5.56 \times 10^{-3}$ & 4.875 & $1.04 \times 10^{-2}$ \\
2.500 & $5.57 \times 10^{-3}$ & 5 & $1.07 \times 10^{-2}$ \\
\hline
\end{tabular}

Example 3. Consider the Fredholm integrodifferential equation:

$$
\begin{gathered}
x^{\prime}(t)=-e^{-t}+\frac{1}{10}\left(-2+e^{-2}+\cos (2)\right)+\cos (t) \\
+\int_{0}^{2} \frac{x(s)}{10} d s, \\
x(0)=1,
\end{gathered}
$$

whose exact solution is $x(t)=e^{-t}+\sin (t)$. The numerical results are given in Table 3 .

Example 4. Consider now the following system of Fredholm integrodifferential equations with the exact solutions $x_{1}(t)=$ $\cos (t)$ and $x_{2}(t)=t$ :

$$
\begin{aligned}
x_{1}^{\prime}(t)= & -\frac{1}{12}-\frac{1}{5} t^{2} \sin (1) x_{2}(s)-\sin (t) \\
& +\int_{0}^{1}\left(\frac{t^{3}}{5} x_{1}(s)+\frac{s^{2}}{3} x_{2}(s)\right) d s,
\end{aligned}
$$


TABLE 3: Absolute errors for Example 3.

\begin{tabular}{lccc}
\hline$t$ & $j=33$ & $t$ & $j=33$ \\
$\left|x_{5}(t)-x(t)\right|$ & 1.125 & $7.05 \times 10^{-4}$ \\
0.125 & $1.00 \times 10^{-4}$ & 1.250 & $7.54 \times 10^{-4}$ \\
0.250 & $1.95 \times 10^{-4}$ & 1.375 & $7.97 \times 10^{-4}$ \\
0.375 & $2.85 \times 10^{-4}$ & 1.500 & $8.34 \times 10^{-4}$ \\
0.500 & $3.69 \times 10^{-4}$ & 1.625 & $8.64 \times 10^{-4}$ \\
0.625 & $4.48 \times 10^{-4}$ & 1.750 & $8.88 \times 10^{-4}$ \\
0.750 & $5.21 \times 10^{-4}$ & 1.875 & $9.06 \times 10^{-4}$ \\
0.825 & $5.89 \times 10^{-4}$ & 2 & $9.19 \times 10^{-4}$ \\
1.00 & $6.50 \times 10^{-4}$ & & \\
\hline
\end{tabular}

TABle 4: Absolute errors for Example 4.

\begin{tabular}{lcccccc}
\hline$t$ & $\begin{array}{c}j=9 \\
\left|x_{51}(t)-x_{1}(t)\right|\end{array}$ & $\begin{array}{c}\mathbf{X}_{5}=\left[x_{51}, x_{52}\right]^{T} \\
\left|x_{52}(t)-x_{2}(t)\right|\end{array}$ & $\begin{array}{c}j=17 \\
\left|x_{51}(t)-x_{1}(t)\right|\end{array}$ & $\begin{array}{c}\mathbf{X}_{5}=\left[x_{51}, x_{52}\right]^{T} \\
\left|x_{52}(t)-x_{2}(t)\right|\end{array}$ & $\begin{array}{c}j=33 \\
\left|x_{51}(t)-x_{1}(t)\right|\end{array}$ & $\begin{array}{c}\mathbf{X}_{5}=\left[x_{51}, x_{52}\right]^{T} \\
\left|x_{52}(t)-x_{2}(t)\right|\end{array}$ \\
\hline 0.125 & $1.7 E-4$ & $4.8 E-5$ & $4.4 E-5$ & $1.2 E-5$ & $1.1 E-5$ & $3.2 E-6$ \\
0.250 & $3.7 E-4$ & $9.7 E-5$ & $9.3 E-5$ & $2.4 E-5$ & $2.3 E-5$ & $6.3 E-6$ \\
0.375 & $5.9 E-4$ & $1.4 E-4$ & $1.4 E-4$ & $3.6 E-5$ & $3.7 E-5$ & $5.5 E-6$ \\
0.5 & $8.2 E-4$ & $1.9 E-4$ & $2.0 E-4$ & $4.8 E-5$ & $5.2 E-5$ & $1.2 E-5$ \\
0.625 & $1.0 E-3$ & $2.3 E-4$ & $2.7 E-4$ & $5.9 E-5$ & $6.8 E-5$ & $1.5 E-5$ \\
0.750 & $1.3 E-3$ & $2.7 E-4$ & $3.3 E-4$ & $7.0 E-5$ & $8.5 E-5$ & $1.0 E-4$ \\
0.875 & $1.6 E-3$ & $3.1 E-4$ & $4.0 E-4$ & $8.0 E-5$ & $2.0 E-5$ \\
1 & $1.9 E-3$ & $3.5 E-4$ & $4.7 E-4$ & $8.9 E-5$ & $1.2 E-4$ & $2.3 E-5$ \\
\hline
\end{tabular}

$$
\begin{gathered}
x_{2}^{\prime}(t)=\frac{20}{21}-\frac{1}{5} t x_{2}(t) \sin (1) \\
+\int_{0}^{1}\left(\frac{t^{2}}{5} x_{1}(s)+\frac{s}{7} x_{2}(s)\right) d s, \\
x_{1}(0)=1, \\
x_{2}(0)=0 .
\end{gathered}
$$

The numerical results are given in Table 4.

Example 5. Consider now the following system of Fredholm integrodifferential equations with the exact solutions $x_{1}(t)=$ $t^{2}$ and $x_{2}(t)=t$ :

$$
\begin{gathered}
x_{1}^{\prime}(t)=2 t-\frac{1}{5} x_{1}(t)(-\cos (1)+\sin (t)) \\
+\int_{0}^{1} \frac{t^{2} s}{5} \sin \left(x_{2}(s)\right) d s, \\
x_{2}^{\prime}(t)=1-\frac{1}{48} x_{2}(t)(\pi-\log (4))+\int_{0}^{1} \frac{t s}{6} \operatorname{arctg}\left(x_{1}(s)\right) d s, \\
x_{1}(0)=0, \\
x_{2}(0)=0 .
\end{gathered}
$$

The numerical results are given in Table 5.
Example 6. Consider now the following system of Fredholm integrodifferential equations with the exact solutions $x_{1}(t)=$ $\sin (t)$ and $x_{2}(t)=\cos (t)$ :

$$
\begin{gathered}
x_{1}^{\prime}(t)=x_{2}(t)+\frac{1}{10}(-1+\cos (2)-\sin (2)) \\
+\frac{1}{10} \int_{0}^{2}\left(x_{1}(s)+x_{2}(s)\right) d s, \\
x_{2}^{\prime}(t)=-x_{1}(t)+\frac{1}{15}(1-\cos (2)-\sin (2)) \\
+\frac{1}{15} \int_{0}^{2}\left(x_{2}(s)-x_{1}(s)\right) d s, \\
x_{1}(0)=0, \\
x_{2}(0)=1 .
\end{gathered}
$$

The numerical results are given in Table 6.

\section{Conclusion}

In this paper we have successfully approximated the solution of systems of nonlinear Fredholm integrodifferential equations. To this end, we have used the Banach fixed-point theorem and the Schauder basis. Moreover, the convergence of the proposed scheme is analyzed and some illustrative examples were included to demonstrate the validity and applicability of the method. The approximating functions $x_{r}$ and $\left[x_{r 1}, x_{r 2}\right]^{T}$ are the sum of integrals of piecewise univariate 
TABle 5: Absolute errors for Example 5.

\begin{tabular}{|c|c|c|c|c|c|c|}
\hline$t$ & $\begin{array}{c}j=9 \\
\left|x_{41}(t)-x_{1}(t)\right|\end{array}$ & $\begin{array}{c}\mathbf{X}_{4}=\left[x_{41}, x_{42}\right]^{T} \\
\left|x_{42}(t)-x_{2}(t)\right|\end{array}$ & $\begin{array}{c}j=17 \\
\left|x_{41}(t)-x_{1}(t)\right|\end{array}$ & $\begin{array}{c}\mathbf{X}_{4}=\left[x_{41}, x_{42}\right]^{T} \\
\left|x_{42}(t)-x_{2}(t)\right|\end{array}$ & $\begin{array}{c}j=33 \\
\left|x_{41}(t)-x_{1}(t)\right|\end{array}$ & $\begin{array}{c}\mathbf{X}_{4}=\left[x_{41}, x_{42}\right]^{T} \\
\left|x_{42}(t)-x_{2}(t)\right|\end{array}$ \\
\hline 0.125 & $3.4 E-7$ & $3.1 E-6$ & $6.1 E-8$ & $8.1 E-7$ & $1.1 E-8$ & $2.3 E-7$ \\
\hline 0.250 & $2.1 E-6$ & $1.2 E-5$ & $4.4 E-7$ & $3.2 E-6$ & $7.3 E-8$ & $9.4 E-7$ \\
\hline 0.375 & $6.5 E-6$ & $2.7 E-5$ & $1.4 E-6$ & $7.2 E-6$ & $2.2 E-7$ & $2.1 E-6$ \\
\hline 0.5 & $1.5 E-5$ & $4.9 E-5$ & $3.3 E-6$ & $1.3 E-5$ & $4.8 E-7$ & $3.9 E-6$ \\
\hline 0.625 & $2.9 E-5$ & $7.7 E-5$ & $6.3 E-6$ & $2.1 E-5$ & $8.3 E-7$ & $6.2 E-6$ \\
\hline 0.750 & $4.9 E-5$ & $1.1 E-4$ & $1.1 E-5$ & $2.9 E-5$ & $1.2 E-6$ & $9.2 E-6$ \\
\hline 0.875 & $7.7 E-5$ & $1.5 E-4$ & $1.6 E-5$ & $4.1 E-5$ & $1.5 E-6$ & $1.2 E-5$ \\
\hline 1 & $1.1 E-4$ & $1.9 E-4$ & $2.4 E-5$ & $5.3 E-5$ & $1.6 E-6$ & $1.7 E-5$ \\
\hline
\end{tabular}

TABLE 6: Absolute errors for Example 6.

\begin{tabular}{|c|c|c|c|c|c|c|}
\hline$t$ & $\begin{array}{c}j=17 \\
\left|x_{31}(t)-x_{1}(t)\right|\end{array}$ & $\begin{array}{c}\mathbf{X}_{3}=\left[x_{31}, x_{32}\right]^{T} \\
\left|x_{32}(t)-x_{2}(t)\right|\end{array}$ & $\begin{array}{c}j=33 \\
\left|x_{31}(t)-x_{1}(t)\right|\end{array}$ & $\begin{array}{c}\mathbf{X}_{3}=\left[x_{31}, x_{32}\right]^{T} \\
\left|x_{32}(t)-x_{2}(t)\right|\end{array}$ & $\begin{array}{c}j=65 \\
\left|x_{31}(t)-x_{1}(t)\right|\end{array}$ & $\begin{array}{c}\mathbf{X}_{3}=\left[x_{31}, x_{32}\right]^{T} \\
\left|x_{32}(t)-x_{2}(t)\right|\end{array}$ \\
\hline 0.125 & $1.1 E-3$ & $4.8 E-5$ & $8.5 E-4$ & $8.3 E-6$ & $9.3 E-5$ & $9.8 E-7$ \\
\hline 0.250 & $2.2 E-3$ & $1.1 E-4$ & $9.2 E-4$ & $2.1 E-5$ & $1.8 E-4$ & $1.1 E-6$ \\
\hline 0.375 & $3.4 E-3$ & $2.0 E-4$ & $2.9 E-3$ & $4.0 E-5$ & $3.3 E-4$ & $1.5 E-6$ \\
\hline 0.5 & $4.5 E-3$ & $3.1 E-4$ & $4.1 E-3$ & $6.3 E-5$ & $5.4 E-4$ & $1.8 E-6$ \\
\hline 0.625 & $5.7 E-3$ & $4.3 E-4$ & $5.6 E-3$ & $9.0 E-5$ & $7.8 E-4$ & $4.3 E-6$ \\
\hline 0.750 & $6.8 E-3$ & $5.7 E-4$ & $6.7 E-3$ & $1.2 E-4$ & $9.2 E-4$ & $8.5 E-6$ \\
\hline 0.875 & $8.0 E-3$ & $7.3 E-4$ & $7.9 E-3$ & $1.5 E-4$ & $3.5 E-3$ & $1.3 E-5$ \\
\hline 1 & $9.2 E-3$ & $9.0 E-4$ & $8.8 E-3$ & $1.9 E-4$ & $5.3 E-3$ & $1.9 E-5$ \\
\hline 1.125 & $1.0 E-2$ & $1.0 E-3$ & $9.7 E-3$ & $2.3 E-4$ & $7.9 E-3$ & $2.6 E-5$ \\
\hline 1.250 & $1.1 E-2$ & $1.2 E-3$ & $1.0 E-2$ & $2.8 E-4$ & $8.2 E-3$ & $3.3 E-5$ \\
\hline 1.375 & $1.2 E-2$ & $1.4 E-3$ & $1.1 E-2$ & $3.2 E-4$ & $9.0 E-3$ & $4.1 E-5$ \\
\hline 1.5 & $1.4 E-2$ & $1.6 E-3$ & $1.3 E-2$ & $3.7 E-4$ & $9.9 E-3$ & $4.9 E-5$ \\
\hline 1.625 & $1.5 E-2$ & $1.8 E-3$ & $1.5 E-2$ & $4.1 E-4$ & $1.0 E-2$ & $5.7 E-5$ \\
\hline 1.750 & $1.6 E-2$ & $2.0 E-3$ & $1.6 E-2$ & $4.6 E-4$ & $1.2 E-2$ & $6.4 E-5$ \\
\hline 1.875 & $1.8 E-2$ & $2.2 E-3$ & $1.7 E-2$ & $5.1 E-4$ & $1.5 E-2$ & $7.2 E-5$ \\
\hline 2 & $1.9 E-2$ & $2.4 E-3$ & $1.8 E-2$ & $5.5 E-4$ & $1.7 E-2$ & $7.9 E-5$ \\
\hline
\end{tabular}

and bivariate polynomials of degree 2 and the calculation of the coefficients of such polynomials just requires linear combinations of several evaluations of the basic functions at sufficient number of points.

\section{Conflict of Interests}

The authors declare that there is no conflict of interests regarding the publication of this paper.

\section{Acknowledgments}

This research is partially supported by Junta de Andalucía Grant FQM359 and the ETSIE of the University of Granada, Spain.

\section{References}

[1] H. Danfu and S. Xufeng, "Numerical solution of integrodifferential equations by using CAS wavelet operational matrix of integration," Applied Mathematics and Computation, vol. 194, no. 2, pp. 460-466, 2007.
[2] A. Jafarian and S. Measoomy Nia, "Utilizing feed-back neural network approach for solving linear Fredholm integral equations system," Applied Mathematical Modelling, vol. 37, no. 7, pp. 5027-5038, 2013.

[3] K. Maleknejad, F. Mirzaee, and S. Abbasbandy, "Solving linear integro-differential equations system by using rationalized Haar functions method," Applied Mathematics and Computation, vol. 155, no. 2, pp. 317-328, 2004.

[4] K. Maleknejad and M. Tavassoli Kajani, "Solving linear integrodifferential equation system by Galerkin methods with hydrid functions," Applied Mathematics and Computation, vol. 159, no. 3, pp. 603-612, 2004.

[5] A. Pedas and E. Tamme, "A discrete collocation method for Fredholm integro-differential equations with weakly singular kernels," Applied Numerical Mathematics, vol. 61, no. 6, pp. 738751, 2011.

[6] J. Pour-Mahmoud, M. Y. Rahimi-Ardabili, and S. Shahmorad, "Numerical solution of the system of Fredholm integrodifferential equations by the Tau method," Applied Mathematics and Computation, vol. 168, no. 1, pp. 465-478, 2005.

[7] S. Yalçinbaş, M. Sezer, and H. H. Sorkun, "Legendre polynomial solutions of high-order linear Fredholm integro-differential 
equations," Applied Mathematics and Computation, vol. 210, no. 2, pp. 334-349, 2009.

[8] E. Yusufoğlu, "Numerical solving initial value problem for Fredholm type linear integro-differential equation system," Journal of the Franklin Institute, vol. 346, no. 6, pp. 636-649, 2009.

[9] Ş. Yüzbaşı, N. Şahin, and M. Sezer, "Numerical solutions of systems of linear Fredholm integro-differential equations with Bessel polynomial bases," Computers \& Mathematics with Applications, vol. 61, no. 10, pp. 3079-3096, 2011.

[10] M. Zarebnia and M. G. Ali Abadi, "Numerical solution of system of nonlinear second-order integro-differential equations," Computers \& Mathematics with Applications, vol. 60, no. 3, pp. 591-601, 2010.

[11] M. I. Berenguer, M. A. Fortes, A. I. Garralda Guillem, and M. Ruiz Galán, "Linear Volterra integro-differential equation and Schauder bases," Applied Mathematics and Computation, vol. 159, no. 2, pp. 495-507, 2004.

[12] M. I. Berenguer, D. Gámez, A. I. Garralda-Guillem, M. R. Galán, and M. C. S. Pérez, "Analytical techniques for a numerical solution of the linear Volterra integral equation of the second kind," Abstract and Applied Analysis, vol. 2009, Article ID 149367, 12 pages, 2009.

[13] M. I. Berenguer, D. Gámez, A. I. Garralda-Guillem, and M. C. Serrano Pérez, "Nonlinear Volterra integral equation of the second kind and biorthogonal systems," Abstract and Applied Analysis, vol. 2010, Article ID 135216, 11 pages, 2010.

[14] M. I. Berenguer, M. V. F. Muñoz, A. I. Garralda-Guillem, and M. R. Galán, "A sequential approach for solving the Fredholm integro-differential equation," Applied Numerical Mathematics, vol. 62 , no. 4 , pp. 297-304, 2012.

[15] G. J. O. Jameson, Topology and Normed Spaces, Chapman \& Hall, London, UK, 1974.

[16] B. R. Gelbaum and J. Gil de Lamadrid, "Bases of tensor products of Banach spaces," Pacific Journal of Mathematics, vol. 11, pp. 1281-1286, 1961.

[17] Z. Semadeni, "Product Schauder bases and approximation with nodes in spaces of continuous functions," Bulletin de l'Académie Polonaise des Sciences, vol. 11, pp. 387-391, 1963. 


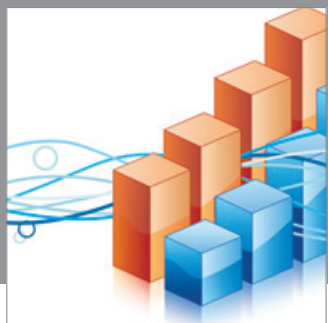

Advances in

Operations Research

mansans

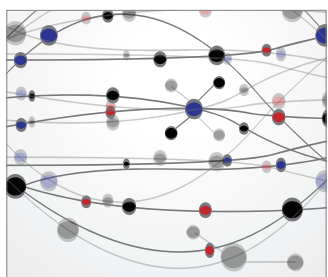

The Scientific World Journal
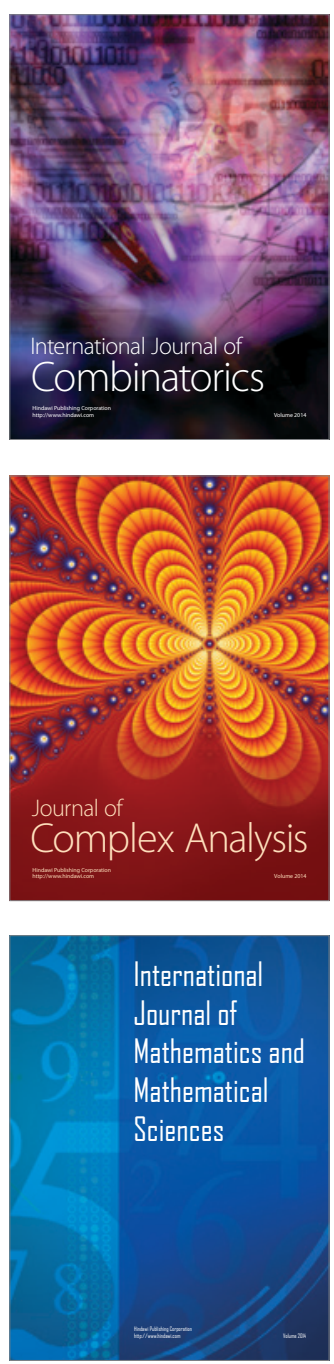
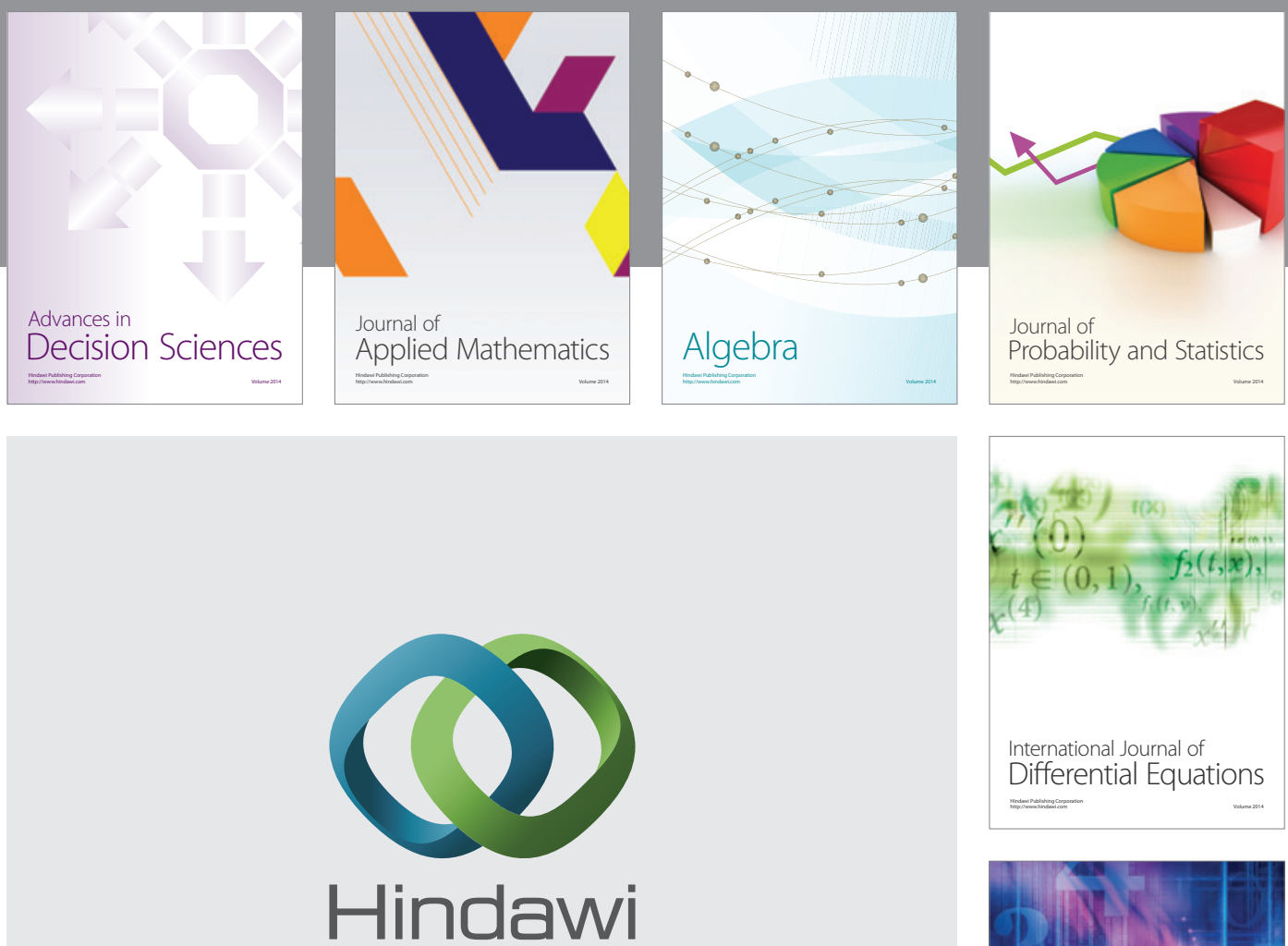

Submit your manuscripts at http://www.hindawi.com
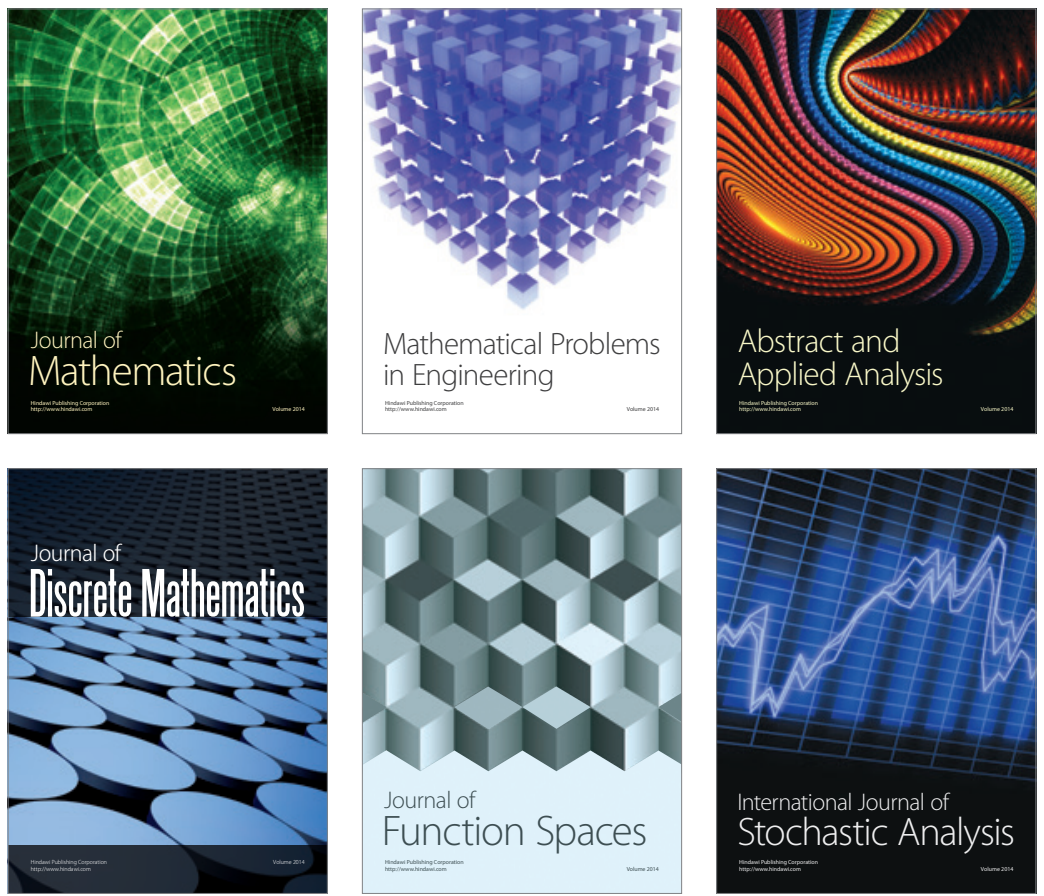

Journal of

Function Spaces

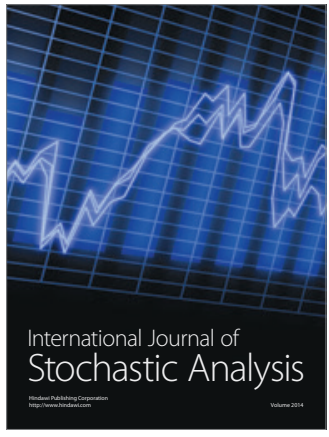

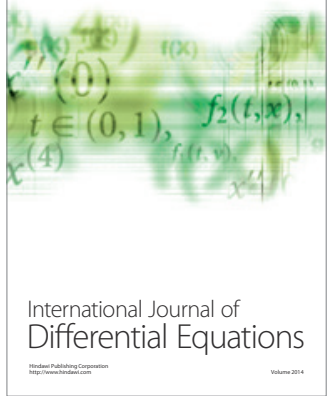
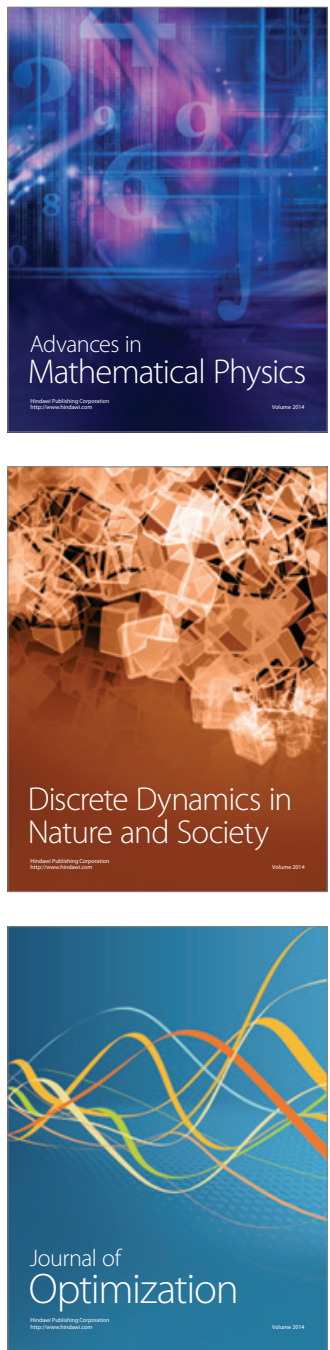